\title{
Nitration of Aromatic Compounds on Silica Sulfuric Acid
}

\author{
Mohammad Ali Zolfigol, ${ }^{, *}{ }^{*}$ BiBi Fatemel Mirjalili, ${ }^{\ddagger}$ Abdolhamid Bamoniri, \\ Mohammad Ali Karimi Zarchi, Amin Zarei, Leila Khazdooz, and Jalil Noci \\ ${ }^{\dagger}$ Chemistry Department, College of Science. Bu-Ali Sina University. Hamadan 65174, P. O. Box 4135, Iran \\ *Department of Chemistry, College of Science, Yazd University, Yazd, P. O. Box 89195-741. Iran \\ Department of Chemistry, College of Science. Kashan Unversity; Kashan 51167. Iram \\ Received November 12. 2003
}

Key Words : Silica sulfuric acid, Sodium nitrate, Nitration, Solvent free conditions

Nitration of organic compounds has long been a very active and rewarding area of rescarch and is the subject of a large body of literature. ${ }^{1}$ Also nitro-aromatics compounds are extensively utilised and act as chemical feedstocks for a wide range of uselul materials such as dyes, pharmaceuticals. perfumes, and plastics. ${ }^{2}$ Therefore, the nitration of aromatic rings has received considerable attention of late, due to unsolved problems pertaining to regioselectivity, overnitration and competitive oxidation of substrates. Nitration of phenol taken as a special case has been studied by various nitrating agents under diflerent conditions. ${ }^{*}$ The nitration of benzene and toluene is one of the most important routs to substituted aromatics in the production of chemical intemediates. 5 The industrial nitration of toluene is performed by means of mixed acid, a mixture of nitric acid, sulluric acid, and water ${ }^{\text {sa }}$ which leading to excessive acid waste streams and added expense. ${ }^{5 b} \mathrm{Also}$, the separation of the products from the acid is olien a difficult and energy consuming process that habitually implies a basic aqueous work-up. Moreover, sulfuric acid is comosive and is dangerous to transport and handle. The above mentioned disadvantages of the commercial manulacturing process currently used has led to a substantial eflort to develop viable altematives, inter idea using solid acid catalyst, $1.2 .-8$ other sources of $\mathrm{NO}_{2}, 3.911$ organic nitrating agents, 11.12 metal nitrates, ${ }^{13.14}$ other acids replacing sulfuric acid such as inorganic acidic salts $\left(\mathrm{NaIHSO}_{4} \cdot\right]_{2} \mathrm{O}, \mathrm{Mg}\left(\mathrm{HSO}_{4}\right), \mathrm{O}$ onone ${ }^{\mathrm{k}}$, $\ldots)^{15}$ and silica sulfuric acid, ${ }^{6.16} \mathrm{etc}$.

Our goal, in undertaking this line of work, was threc-lold: a) 10 overcome the limitations and drawbacks of the reported methods such as tedious work-up. ${ }^{17.18}$ strongly acidic media. ${ }^{13}$ oxidation ability of the reagents and salety problems (storage. handling, using and also presence of toxic transition metal cations such as $\mathrm{Cr}^{3}, \mathrm{HIg}^{12}, \mathrm{Cu}^{12} \ldots$ within molecular structure of the reagents), ${ }^{13.14}$ (b) to perform solvent-liee organic synthesis which seems to be a highly useful technique, especially for industry possessing many advantages like: reduced pollution. low costs, as well as simplicity in process and handling (these factors are especially important in industry), ${ }^{19}$ (c) moreover, to develop an high-yiclding onepol synthesis of nitro-aromatics using a novel combination

"Corresponding Author. e-mail: Zolligatbasuac.ir. Fax: +98-8118272404 of reagents.

In addition, any reduction in the amount of liquid acids needed and/or any simplification in handling procedures would be highly convenient in terms of risk control, economic advantage and environment protection. ${ }^{\text {lis }}$ Recently, we have reported the preparation of silica sulfuric acid I as a stable acidic reagent (solid acid) and showed its ealalytic activity in synthetic methodology. ${ }^{16.21}$ In continuation of our studies in this regard, we have lound that aromatic rings can be nitrated by using a combination of silica sulfuric acid $\mathbf{I}$, $\mathrm{NaNO}_{3}$ and wet $\mathrm{SiO}_{2}$ under solvent free conditions. Therefore, we wish to report here a one-pot solid phase procedure for the nitration of different kind of aromatic compounds.

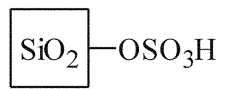

I

$A$ good range of aromatic compounds 1 were also subjected to nitration in the presence of silica sulfurte acid $\mathbf{I}$, $\mathrm{NaNO}_{3}$, and wet $\mathrm{SiO}_{2}(60 \%$ w/w) under solvent free conditions (Scheme 1). The nitration reactions were performed under mild conditions with moderate to good yiclds (Scheme 1 and Table) by simply placing the nitrating agents and substrates 1 in a reaction vessel and efficiently shaking the resulting mixlure. llighly pure nitro compounds can be obtained by simple extraction and subsequent evaporation of the solvent. For this new system the in-situ generation of nitronium ion mechanism may be proposed (See Schemes 2 and 3 ).

\section{Experimental Section}

General. Chemicals were purchased from Fluka, Merck and Aldrich chemical companies. Yields reler to isolated

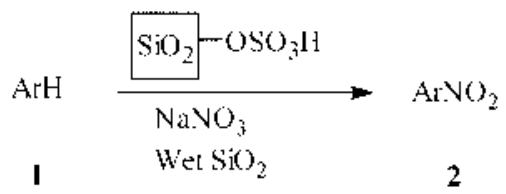

Scheme I 


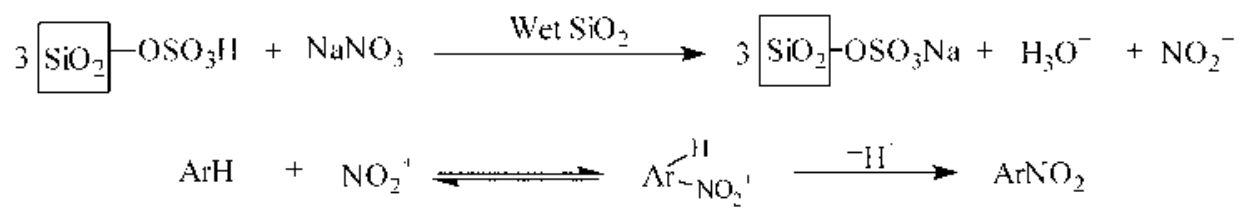

Scheme 2

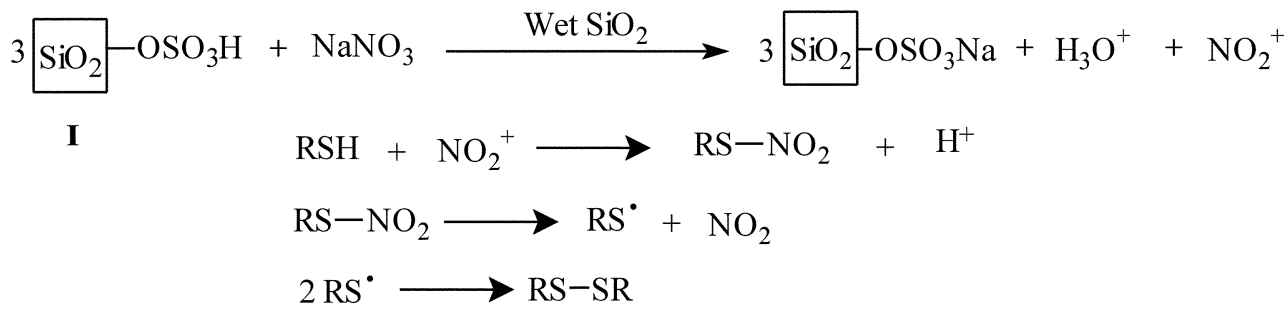

Scheme 3

Table 1. Nitration of aromatic rings with siliea sulfuric acid (I). sodium nitrate. and $w e t \mathrm{SiO}_{2}(60 \% \mathrm{w} / \mathrm{w})$ under solvent free condition

\begin{tabular}{|c|c|c|c|c|c|c|c|}
\hline \multirow{2}{*}{ Entry } & \multirow{2}{*}{ Substrate } & \multirow{2}{*}{$\begin{array}{c}1 \\
\left.{ }^{\circ} \mathrm{C}\right)\end{array}$} & \multirow{2}{*}{$\begin{array}{l}\text { Time } \\
\text { (mirl) }\end{array}$} & \multirow{2}{*}{$\begin{array}{l}\text { Yield } \\
(\%)\end{array}$} & \multirow{2}{*}{ Product } & \multicolumn{2}{|c|}{$\mathrm{M}_{\Gamma}\left(\mathrm{I}_{\Gamma}\right)^{\circ} \mathrm{C}$} \\
\hline & & & & & & Found & Reported ${ }^{21}$ \\
\hline 1 & Benzent & 25 & $2-3$ & 80 & Nitrobenzens & $(208-210)$ & $(2 \mid 0)$ \\
\hline 2 & Toluene & 25 & $2-3$ & 85 & 4-Nitrotoluen & $53-55$ & 54 \\
\hline 3 & 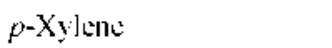 & 25 & $2-3$ & 90 & 2-Nitro-p-xylane & $(232-233)$ & $(234)$ \\
\hline 4 & $0-X y]$ Ln: & 25 & $2-3$ & 85 & 4-Nitro-o-xylenc & $30-31$ & 30 \\
\hline 5 & Thiophenol & 25 & $6-7$ & 90 & Diphenyl disultidd: & $62-64$ & 62 \\
\hline 6 & Phunol & 25 & 12 & 85 & 4-Nitrophenol & $|13-1| 4$ & 114 \\
\hline 7 & Bromobunzene & 25 & $3-4$ & 85 & 4-Nitrobromobenzenc & $125-126$ & 127 \\
\hline 8 & Chlorobenzene & 25 & $3-4$ & 80 & 4-Nitrochlorobenzent & $82-83$ & 83 \\
\hline 9 & Aniso] & 25 & $2-3$ & 85 & 4-Nitroanisol & $52-53$ & 54 \\
\hline 10 & Bipheny] & $60^{r}$ & $30-35$ & 75 & 4-nitrobiphenyl & $|12-1| 3$ & 114 \\
\hline 11 & 3-Chloropheno] & 25 & $10-12$ & 81 & 2-Nitro-5-chlorophenol & $40-43$ & 42 \\
\hline 12 & N.N-Dimethyl aniline & 25 & $\mathrm{I}-2$ & 94 & 4-Nitro- $\mathrm{N}$ - N-dimethy]aniline & $160-163$ & 163 \\
\hline 13 & 4-Methoxy benzaldehyde & 25 & $6-7$ & 74 & 3-Nitro-4-methoxybanzaldelyyde & $83-85$ & 86 \\
\hline 14 & Naphthalene & 50 & 15 & 91 & ]-Nitronaphthalenc & $70-71$ & 71 \\
\hline
\end{tabular}

"was pulverized in a mortar in 25 " $\mathrm{C}$ for I min and then heated until $600^{\circ} \mathrm{C}$ for $30-3.5 \mathrm{~min}$

pure products. The nitration products were characterized by comparison of their spectral (IR. 'H-NMR), TLC and physical data with authentic samples.

General Procedure. A mixture of silica sulfuric acid I $(0.6 \mathrm{~g})$, sodium nitrate ( $\mathrm{l}$ mmol, $0.085 \mathrm{~g})$, wet $\mathrm{SiO}_{2}(0.5 \mathrm{~g}$. $60 \% \mathrm{w} / \mathrm{w}$ ) and aromatic compound $1(\mathrm{l} \mathrm{mmol})$ was pulverized in a mottar for 2-35 min (the progress of the reaction was monitored by TLC), followed by $\mathrm{CH}_{2} \mathrm{Cl}_{2}(10 \mathrm{~mL})$ addition and filtration of the resulting mixture. Dichloromethane was finally removed and the nitro compounds were obtained with moderate to good yields.

IR and ${ }^{1} H-N M R$ of some of the nitrated products.

4-Nitro, N, N-Dimethyl aniline: IR (KBr) 800, 1300, 1350. $1500,1525,1600,2900-3000 \mathrm{~cm}^{-1}:{ }^{1} \mathrm{H}$ NMR $\left(\mathrm{CDCl}_{3}\right) \delta_{\mathrm{ppm}}$ 2.89 (s, 6H), 6.32 (d). 7.8 (d).

p-Nitrophenol: 'H NMR (CDClis) $\delta_{p p m} 6.9$ (d, 2H), 8.1 (d, $2 \mathrm{H}), 10(\mathrm{~s}, 1 \mathrm{H}, \mathrm{O}-\mathrm{H})$.

l-Nitronaphthalene: IR (KBr) $1330,1520,3050 \mathrm{~cm}^{-1}:{ }^{1} \mathrm{H}$ NMR $\left(\mathrm{CDCl}_{3}\right) \delta_{p p m} 8.43(\mathrm{~d}, 1 \mathrm{H}), 7.17-8.1(\mathrm{~m}, 6 \mathrm{H})$.

1-Bromo-4-Nitrobenzene: ${ }^{\mathrm{H}} \mathrm{H}$ NMR $\left(\mathrm{CDCl}_{3}\right) \delta_{\mathrm{ppm}} 7.8(\mathrm{~d}$,
$2 \mathrm{H}), 8.3(\mathrm{~d}, 2 \mathrm{H})$.

2-Nitro-p-xylene: 'H NMR (CDCly) $\delta_{\text {ppm }} 2.4$ (s, 3H), 2.7 (s, 3H), $7.5(\mathrm{~s}, 2 \mathrm{H}), 8.1(\mathrm{~s}, \mathrm{lH})$.

Diphenyl disulfide: 'H NMR (CDClis) $\delta_{p p m} 7.3-7.8(\mathrm{~m}$, $10 \mathrm{H})$ ppm.

3-Nitro-4-methoxy benzaldehyde: 'H NMR (CDCli) $\delta_{\text {ppm }}$ 10 (s, 1H), 8.5 (sbr, lH), 8.2 (dd, IH), 7.3 (dbr, IH), 4.1 (s. $3 \mathrm{H})$.

5-Chloro-2-Nitro phenol: 'H NMR (CDCli) $\delta_{\text {ppm }} 11$ (s. $1 \mathrm{H}, \mathrm{OH}), 8(\mathrm{~d}, 1 \mathrm{H}), 7.6(\mathrm{~d}, 1 \mathrm{H}), 7(\mathrm{dd}, 1 \mathrm{H})$.

4-Nitrobiphenyl: 'H NMR $\left(\mathrm{CDCl}_{3}\right) \delta_{\text {ppm }} 8.5(\mathrm{~d}, 2 \mathrm{H}), 7.8$ (d, 2H). 7.3-7.7 (m, 5H).

Acknowledgement. The authors gratefully acknowledge partial support to this work by the Research Affair of Bu-Ali Sina University, Hamadan, I.R Iran.

\section{References}

1. Min. S.: Shi-Cong. C. J. Fltorine Chem. 2002, //3. 207. 
2. Esakkidurai. T.: Pitchumani. K. J. Mol. Cat. A: Chemical 2002. 185. 305 .

3. Iranpoor. N.: Firouzabadi. H: Heydari. R. Stmh. Commm. 1999. 29.3295 .

4. Zoltigol, M. A. Ghaemi, E.: Madrakian, E. Sinten 2003, 191 and references cited therein.

5. (a) Kogelbauer A. Vassena, D: Prins, R.: Amor J. N. Catalysis Today 2000. 55. 151. (b) Dagade. S. P.: Waghmode. S. B.: Kadam. V. S.: Dongare. M. K. Applied Catalysis A 2002. 226. 49.

6. Riego. J. M.: Sedin. Z.: Zaldivar. J. M.: Marziano. N. C.: Tortato. C. Tetrahedron Lett. 1996.37, 513.

7. Peng, X.: Suzuki. H.: LU, C. Tetrahedron Lett $2001,42.4357$.

8. Dagade. S. P.: Waghmode, S. B.: Kadam, V. S.: Dongare, M. K. Applied Catalysis A 2002. 226.49.

9. Radoslaw. R. B.: Andrew J. S. Tetrahedron Lett. 2001. 42.6767.

10. Iranpeor. N.: Firouzabadi. H.: Heydari. R. Phosphorts, Sulfur and Siticon $2003,178,1027$.

11. Rodrigues. J. A. R.: Oliveira Filho, A. P.: Moran, P. J. S.: Custodio. R. Tetrahedron 1999.55,6733.

12. Rodrigues. J. A. R.: Oliveira Filho. A. P.: Moran. P. J. S. Simth. Conmin. 1999. 29.2169.

13. (a) Delaude. L.: Laszlo. P.: Smith. K. Acc. Chem. Res. 1993. 26. 607. (b) Laszlo. P. Acc. Chen. Res. 1986. 19. 121. (c) Connelis. A.: Laszlo. P. Pennetreau, P. Bull. Soc. Chim. Belg. 1984. 93, 961.

14. (a) Zolfigol. M. A.: Iranpoor. N.: Firouzabadi, H. Oriem. J. Chem. 1998. 1H. 369. (b) Firouzabadi. H.: Iranpoor. N.: Zolfigol. M. A. Iran. J. Chem \& Chem. Eng. 1997, 16. 48. (c) Firouzabadi. H.: Iranpeor. N.: Zoltigol. M. A. Sinth. Commm 1997, 27.3301. (d) Iranpoor, N.: Firouzabadi. H.: Zolfigol. M. A. Sinh Conmun.
1998. 28,2773

15. (a) Zolfigol. M. A.: Bagherzadeh. M.: Madrakiant. E.: Ghaemi. E.: Taqian-t1asab. A. J. Chent. Res(S). 2001. 140. (b) Zoltigol. M. A.: Madrakian. E.: Ghaemi. E. Indian J. Chent 2001. 408.1191.

16. (a) Zolfigol, M. A.; Ghaemi, E.; Madrakian, E. Molecules 2001, 6. 614. (b) Zolfigol, M. A.; Ghaemi. E.: Madrakian, E. Molecules 2002. 7,734 .

17. Crivello. T. V. J. Org. Chem. 1981. 46.3056.

18. Poirier. J. M.: Vottero. C. Tetrohedron 1989. 45. 1415.

19. (a) Tanakia. K.: Toda. F. Chent. Rev 20(00. 100. 1025. (b) Krchnak. V.: Holladay. M. W. Chem. Ren: 2002, 102.61. (c) Varma. R. S. Green Chem. 1999. 1.43.

20. (a) Zolfigol. M. A. Tetahedron 2001, 57.9509. (b) Mirjalili. B. F.; Zolfigol. M. A.: Bamoniri. A. J. Kowam Chem. Soc. 2001. 45. 546. (c) Zolfigol. M. A.: Bamoniri. A. Sintet 2002. 1621 . (d) Mirjalili. B. F.: Zolfigol. M. A.: Bamoniri. A. Molecules 2002. 7. 751. (e) Zolfigol, M. A.; Shirini. F.: Ghorbani-Choghamarani. A.: Mohammadpoor-Baltork, I. Green Chem. 2002, +, 562. (f) Salehi. P.: Dabiri, M.: Zolfigol, M. A.: Bodaghi-fard. M. A. Tetrahedron Lett. 2003. 41. 2889. (g) Zolfigol. M. A.: Chehardoli. G. A.: Mallakpour. S. E. Smth. Conmmm. 2003. 33. 833. (h) Mirjalili. B. F.: Zolfigol. M. A.: Bamoniri. A.: Zarei. A. Bull. Korem Chent. Soc. 2003, 24. 400. (i) Zolfigol, M. A.; Ghorbani-Choghamarani. A. Phosphorus. Sulfur and Silicon 2003, 178, 1623. (j) Shirini. F.: Zolfigol, M. A,; Mohammadi, K. Phosphorus. Sulfur and Silicon 2003. 178. 1617. (k) Mijalili. B. F.: Zoltigol. M. A.: Bamoniri. A.: Zarei. A. Phosphorus, Sulfur and Silicon 2003. 178. 1845.

21. Dictionay of Orgamic Conpomds. 3th Ed: Eyre and Spottiswoode: London. 1965. 